\title{
Plädoyer für eine rational begründete restriktive Indikation zur Bluttransfusion
}

\author{
Georg Geissler, Clemens Kösters, Walter Sibrowski, Michael J. Raschke, Peter Schlenke
}

\section{Zusammenfassung}

Die demografische Entwicklung in Europa und Deutschland lässt erwarten, dass der zukünftige Blutbedarf potenziell zunehmen wird. Dies ist zum einen dem medizinischen Fortschritt und der daraus resultierenden höheren Lebenserwartung geschuldet, zum anderen dadurch begründet, dass ältere Patienten wegen ihrer Grunderkrankungen und Komorbiditäten erfahrungsgemäß wesentlich mehr Transfusionen benötigen als jüngere Patienten. Die Zahl freiwilliger gesunder Blutspenderinnen und Blutspender wird hingegen in den nächsten Jahren wegen der nachrückenden geburtenschwachen Jahrgänge überproportional abnehmen; zudem könnte sich ihre langfristige Bindung an Blutspendeeinrichtungen als schwierig erweisen. Die sich abzeichnende Verknappung der natürlichen Ressource „Blut" erfordert einen sehr sorgfältigen und verantwortungsvollen therapeutischen Umgang mit Blutkomponenten, wie ihn die Bundesärztekammer in den aktuellen Querschnittsleitlinien zur Therapie mit Blutkomponenten und Plasmaderivaten fordert.

Genau hier setzen sog. Patient Blood Management (PBM)-Konzepte an, die in Australien, Österreich und der Schweiz ihren Ursprung genommen haben und heute weltweit klinische Beachtung finden. PBM geht über die Idee einer rational begründeten, risikominimierten Hämotherapie hinaus, indem es auch Strategien zur grundsätzlichen Transfusionsvermeidung einschließlich einer Verbesserung des perioperativen Gerinnungsmanagements entwickelt. Sowohl Konzepte zur rationalen Hämotherapie als auch zum PBM erheben den Anspruch, den jeweils aktuellen Stand von Wissenschaft und Technik nach evidenzbasierten Grundsätzen wiederzugeben. Neuere Ergebnisse mehrerer randomisierter klinischer Studien und Metaanalysen geben Anlass, (selbst-)kritisch liberale Transfusionsstrategien $\mathrm{zu}$ hinterfragen, präventive Maßnahmen zur Vermeidung und Reduktion von Transfusionen zu favorisieren und allogene Transfusionen patientenindividuell erst nach sorgfältiger Nutzen-Risiko-Abwägung zu verordnen.

Dieser Artikel beschreibt die sog. hämotherapeutische Kette, beginnend bei der sorgfältigen Spenderauswahl und Spendertestung, über die Weiterverarbeitung von freiwillig geleisteten Blutspenden zu qualitativ hochwertigen Blutprodukten bis hin zu deren optimierter klinischer Anwendung. Hierbei wird auch auf Erfahrungen aus der im Jahr 2012 gegründeten PBMInitiative am Universitätsklinikum Münster (UKM) eingegangen. Im Alltag orthopädisch-unfallchirurgischer Kliniken besteht der größte Bedarf an Blutprodukten in den Bereichen Polytraumaversorgung, Endoprothetik sowie Osteosynthese der langen Röhrenknochen und am Becken. In der Polytraumaversorgung unterliegt die Indikation zur Transfusion einer interdisziplinären Entscheidungsfindung meist in der frühen Versorgungsphase, d.h. im Schockraum, im Operationssaal oder auf der Intensivstation. Auch bei elektiven Eingriffen sollten krankenhausinterne Bereitstellungs- und Transfusionsalgorithmen unter Berücksichtigung der Vorgaben aus den aktuellen Querschnittsleitlinien implementiert werden.
A Plea for Rationally Based Restrictive Indication for Blood Transfusion

The demographic developments in Europe and Germany suggest that the future need for blood products is likely to increase. On the one hand this may be due to medical progress and the resulting increase in life expectancy, on the other hand due to the experience that because of their underlying diseases and comorbidities older patients need significantly more transfusions than younger patients. Because of the low birth rates, the number of voluntary healthy blood donors may decrease disproportionately in the near future. In addition, the long-term commitment of new blood donors to blood donation centres could prove difficult. This emerging shortage of the natural resource "blood" requires a very careful and responsible therapeutic use of blood components, as that which the German Medical Association calls for in the current cross-sectional guidelines for therapy with blood components and plasma derivatives.

For this very reason "patient blood management" (PBM) concepts have found their origins in Australia, Austria and Switzerland and are now attracting clinical attention worldwide. By developing strategies for the basic avoidance of transfusions, including an improvement of perioperative coagulation management, PBM goes beyond the idea of a rationally based, risk-minimised haemotherapy. Both approaches to rational haemotherapy and to PBM claim to reflect the current state of science and technology with evidencebased principles. Recent results of several randomised clinical trials and 
meta-analyses give reason to question liberal transfusion strategies (self-)critically, to favour preventive measures to avoid and reduce transfusions, and to prescribe patient-specific allogeneic transfusions only after careful benefitrisk assessments.

This article discusses the so-called transfusional therapeutic chain, beginning with the careful selection of donors and donor testing, continuing with the re-processing of voluntary donated blood to attain high-quality blood products for an optimised clinical application. The text also discusses experience gained from the PBM initiative founded in 2012 at the University Hospital of Muenster. In the routine of orthopaedic surgical clinics the greatest need for blood products arises in multiple traumas, in arthroplasty and in osteosyntheses of long bones and the pelvis. In multiple trauma patients, decision-making for blood transfusions is the subject of an interdisciplinary cooperation usually in the early phase of therapy, i.e., in the emergency room, in the operating room, or in the intensive care unit. Standard protocols for elective operations referring to indication policies and to the preoperative algorithms for blood products should be implemented taking into account the currently valid guidelines.

\section{Blutbedarf}

Nach Angaben des Paul-Ehrlich-Instituts wurden 2011 in Deutschland über 4,3 Mio. Erythrozytenkonzentrate, etwa 1,1 Mio. Plasmaeinheiten und fast 500000 Thrombozytenkonzentrate verbraucht [1]. Der Bedarf für diese 3 Blutkomponenten ist in den operativen Disziplinen am höchsten für die Herz-Thorax-Gefäß-Chirurgie, gefolgt von der Viszeralchirurgie und der Unfallchirurgie/Orthopädie [2-6]. Dabei steht der Verbrauch allogener Erythrozytenkonzentrate an erster Stelle [5,6] (Abb. 1).

Gemäß einer retrospektiven Analyse in der unfallchirurgischen Klinik am Universitätsklinikum Münster (Geißler et al., in Vorbereitung) erhalten bspw. $>50 \%$ der polytraumatisierten Patienten durchschnittlich 4-6 Erythrozytenkonzentrate und ca. ein Drittel der Patienten mit endoprothetischer Versorgung durchschnittlich 1-2 Erythrozytenkonzentrate. Massivtransfusionen sind selbst in Krankenhäusern der Maximalversorgung und auch im Patientenkollektiv mit Polytraumata eher selten (ca. 10\%).

Obwohl auch durch elektiven Einsatz von Eigenblutspenden die Quote der allogenen Bluttransfusionen, bspw. in der Hüft- und Kniegelenkschirurgie, reduziert werden kann [7], ist diese autologe Blutspende in Deutschland quantitativ nur noch von untergeordneter Bedeutung $(<1 \%)$ [1]. Dies mag dem hohen organisatorischen Aufwand der Eigenblutspende, der sehr hohen Infektionssicherheit von Fremdblut (im Gegensatz zum AIDS-Skandal in den 80er-Jahren), verschiedenen Kontraindikationen zur Eigenblutspende (z.B. präoperative Anämie) und der bekannt hohen Verwurfrate geschuldet sein.

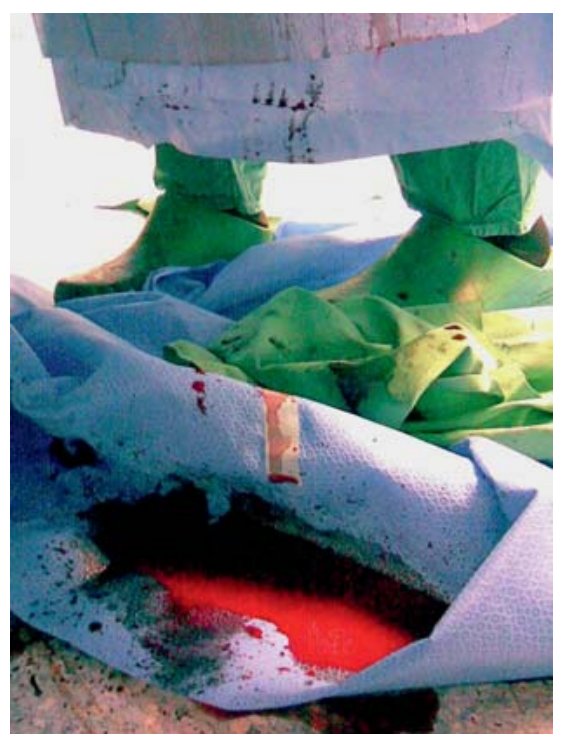

Abb. 1 BOF-Syndrom („blood on floor“). Nicht abschätzbarer Blutverlust bei einem operativen Eingriff (mit freundlicher Genehmigung von Dr. Klaus Görlinger, München).

\section{Demografie}

Wie in den meisten Ländern westlicher Prägung wird auch in Deutschland künftig eine Verknappung der 3 Blutkomponenten erwartet. Dies hat mehrere Ursachen. Der Altersdurchschnitt der Bevölkerung steigt signifikant [8]. Auch werden ältere Patienten durchschnittlich wesentlich häufiger transfundiert als jüngere $[9,10]$. 70 - bis 80 -jährige $\mathrm{Pa}$ tienten benötigen einer finnischen Studie zufolge 8-mal mehr Erythrozytenkonzentrate als 20- bis 40-Jährige [11].

Die verbesserten anästhesiologischen, operativen und intensivmedizinischen Behandlungsoptionen ermöglichen es zudem, vermehrt ältere und schwerkranke Patienten komplexen Operationen zu unterziehen $[9,12,13]$.
Auch nimmt die Zahl organ- und stammzelltransplantierter Patienten zu. All diese Entwicklungen erhöhen prinzipiell den Bedarf an Blutkomponenten [12].

Demgegenüber wird künftig die Zahl der vornehmlich jungen Blutspender demografisch bedingt abnehmen [8-10, $12,14]$.

Hier könnten Steigerungen erzielt werden, da nur ca. 4\% aller Menschen im spendefähigen Alter (18-68 Jahre) Wiederholungsspender sind [15]. Allerdings liegt die aktuelle deutsche „Blutspendefreudigkeit" deutlich über dem europäischen Durchschnitt [16]. Große, flächendeckende Werbekampagnen zeigen ein nur begrenztes Steigerungspotenzial auf. Zudem werden aus Sicht des PBM proaktive Strategien zur Transfusionsvermeidung und restriktive Transfusionsalgorithmen zur Verbesserung des patientoutcome zu befürworten, die sich mittelbar auch auf das (dann noch) notwendige Spendeaufkommen auswirken können.

\section{Hämovigilanz}

Bluttransfusionen sind bei Patienten mit sehr hohem Blutverlust und ausgeprägter erkrankungs- oder therapiebedingter Anämie und daraus resultierender anämischer Hypoxie unbestreitbar lebensrettende Therapien. Sie sind darüber hinaus mit Einschränkung auch medizinisch indiziert, um die Lebensqualität des informierten und aufgeklärten $\mathrm{Pa}$ tienten zu verbessern.

Bluttransfusionen bergen jedoch auch bekannte infektiologische und immunologische Risiken $[17,18]$, die durch sehr differenzierte Vorgaben zu Spenderauswahl und Spendertestung [20-22] mit Verbesserung der Sicherheit für den Empfänger von Blutkomponenten in den letzten Jahrzehnten deutlich reduziert werden konnten [22]. 
Im Jahr 2010 wurden dem Paul-EhrlichInstitut bei etwa 6 Mio. Transfusionen lediglich 702 Transfusionsreaktionen ( $0,01 \%$ ) gemeldet [22]. 100 dieser Fälle waren febrile, nicht hämolytische Reaktionen, die meist keinen schwerwiegenden klinischen Verlauf aufweisen. Auch 219 weitere, zumeist allergische Transfusionsreaktionen wurden als nicht schwerwiegend eingestuft. Somit traten 2010 insgesamt nur 383 schwerwiegende Transfusionsreaktionen auf [22], darunter 110 schwerwiegende allergische Reaktionen, 29 Fälle einer transfusionsbedingten Volumenüberladung (TACO), 18 hämolytische Reaktionen, 4 Reaktionen mit transfusionsassoziierter akuter Lungeninsuffizienz (TRALI), 4 Fehltransfusionen, 3 transfusionsbedingte bakterielle, 2 transfusionsbedingte virale Infektionen und ein Fall einer sog. posttransfusionellen Purpura [22].

Grundsätzlich ist bei der freiwilligen Erhebung nationaler Hämovigilanzdaten, so auch in Deutschland, von einem „underreporting“ auszugehen. Darüber hinaus sind diese genannten schwerwiegenden Transfusionsreaktionen nicht mit dem Phänomen der transfusionsassoziierten Immunmodulation (TRIM) nach Fremdblutgabe gleichzusetzen. TRIM trägt kausal zu einer höheren Morbidität und Mortalität transfundierter Patienten bei. Dies ist gemäß mehrerer klinischer Studien [23,24] unabhängig vom Schweregrad der Erkrankung bzw. Therapie und weiterer vorbestehender Risikofaktoren (z.B. präoperative Anämie) der Fall.

\section{Patient Blood Management}

Die Erkenntnis, dass Transfusionen einen unabhängigen Risikofaktor für einen schlechteren Behandlungserfolg darstellen, ist aktuell Anlass, die medizinische Indikation zur Transfusion von Erythrozytenkonzentraten, aber auch von gefrorenen Frischplasmen und Thrombozytenkonzentraten zu überdenken und PBM-Konzepte zur kontinuierlichen klinischen Qualitätsverbesserung zu implementieren. Letztgenannte Maßnahmen sollen dazu beitragen, „alt bewährte Traditionen “ in der routinemäßigen Patientenversorgung zu durchbrechen und die erheblichen Varianzen im alltäglichen Umgang mit präoperativen Anämien, intraoperativem Blutverlust und der Indikationsstellung zur Transfusion nach den Grundsätzen einer evidenzbasierten Medizin zu vereinheitlichen $[25,26]$.

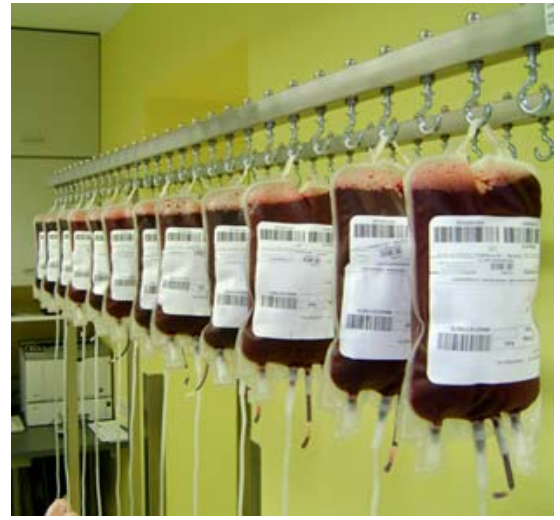

Abb. 2 Filterung von Erythrozytenkonzentraten (mit freundlicher Genehmigung von jürgen Deutschkämer, UKM).

Nachfolgend wird vornehmlich auf den Einsatz von Erythrozytenkonzentraten eingegangen. Ein gutes Beispiel für eine unerwartet hohe, medizinisch kaum begründbare Variabilität im Blutbedarf sind elektive Operationen zum Hüftund Kniegelenksersatz. So wurden zu 15-39\% präoperative Anämien diagnostiziert, und die Substitution mit allogenen Erythrozyten variierte zwischen 9 und 92\% [7]. Patienten mit perioperativen Anämien weisen postoperativ gehäuft Infektionen (Wundgebiet, Harnwege, Lunge), verlängerte stationäre Aufenthalte sowie eine erhöhte Mortalitätsrate auf, wie die Arbeitsgruppe von Spahn publizierte [7]. Ergänzend fanden andere Autoren, dass eine präoperative Anämie generell das Risiko für postoperative Mortalität und Morbidität erhöht [12], so auch an über 69000 (darunter über 2700 unfallchirurgisch-orthopädischen) Patienten mit präoperativer Anämie und nachfolgend nicht kardiochirurgischem Eingriff [27].

Bereits im Jahr 1999 publizierten Hébert et al. die bahnbrechenden Ergebnisse ihrer randomisierten multizentrischen Studie mit einem großen Kollektiv $(\mathrm{n}>800)$ an intensivpflichtigen Patienten [28]. Sie konnten zeigen, dass eine restriktive Strategie mit einem Transfusiontrigger unter $7 \mathrm{~g} / \mathrm{dl}$ Hämoglobin mind. genauso effektiv wie ein liberales Vorgehen mit Transfusionen unter $9 \mathrm{~g} / \mathrm{dl}$, möglicherweise diesem liberaleren Regime sogar überlegen war. Aktuell wurde auch für Patienten mit einem Durchschnittsalter von über 80 Jahren und entsprechendem kardialem, zerebrovaskulärem und sonstigem altersbedingtem Risikoprofil mit Eingriffen in der Hüftgelenkschirurgie gezeigt, dass diese Patienten ebenfalls nicht von einem liberalen

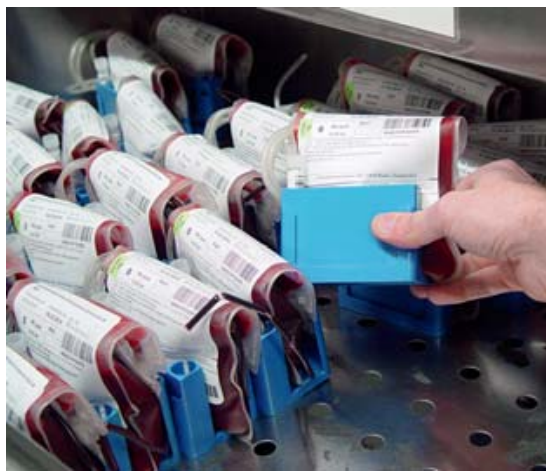

Abb. 3 Blutdepot (mit freundlicher Genehmigung von Dr. Hartmut Hillmann, UKM).

Transfusionsregime mit Erythrozytengaben bei Hämoglobinwerten unter $10 \mathrm{~g} / \mathrm{dl}$ bezüglich Mortalität, Mobilisierungsfähigkeit und kardiovaskulären Risiken profitieren [29]. Daher werden mittlerweile meist restriktive Indikationen zur Erythrozytentransfusion empfohlen und aufgrund der klinischen Tragweite auch in krankenhausinternen Audits überprüft [30]. Eine Ausnahme könnte das akute Koronarsyndrom darstellen.

PBM-Initiativen als Multidisziplinen möchten aufgrund der immunsuppressiven Wirkung allogener Erythrozyten sowohl Chirurgen, Anästhesiologen und Transfusionsmediziner als auch Patienten für die Thematik sensibilisieren und präventive sowie therapeutische Maßnahmen (erfolgreiche Anämiebehandlung, blutsparende Operationstechnik, Ausschöpfung der patientenindividuellen Anämietoleranz) zur Vermeidung von Transfusionen maßgeblich fördern [31-33] (Abb. 2 und 3).

Zu einem vollständigen PBM-Programm gehört präoperativ eine sorgfältige, ggf. internistische Anämieabklärung und eine ebenso sorgfältige Erhebung der Blutungs- bzw. Medikamentenanamnese mit ggf. zu veranlassender Gerinnungsdiagnostik.

Je nach Dringlichkeit des operativen Eingriffs ist eine Anämietherapie (z. B. intravenöse Eisensubstitution) [34] bzw. eine Umstellung der antikoagulativen Behandlung angezeigt und die Verschiebung des Operationstermins in Kauf zu nehmen.

Auch prä- und postoperative Erythropoetingaben zur Reduktion allogener Erythrozytensubstitution werden trotz unerwünschter Wirkungen und hoher Therapiekosten diskutiert, so z.B. bei 
Tab. 1 Die 3 Säulen des Patient Blood Management (nach [18, 32]).

\begin{tabular}{llll}
\hline & $\begin{array}{l}\text { Optimierung der } \\
\text { Erythrozytenzahl }\end{array}$ & Minimierung des Blutverlusts & $\begin{array}{l}\text { Optimierung der physiologischen } \\
\text { Reserven }\end{array}$ \\
\hline präoperativ & $\begin{array}{l}\text { Anämiediagnostik, -thera- } \\
\text { pie, ggf. Eigenblutspende, } \\
\text { ggf. Elektiv-OP verschieben }\end{array}$ & $\begin{array}{l}\text { Blutungsrisiken eruieren (Eigenanamnese, } \\
\text { Medikation, Familienanamnese,), iatrogene } \\
\text { Blutverluste meiden, ggf. OP-Planung indivi- } \\
\text { duell anpassen }\end{array}$ & $\begin{array}{l}\text { restriktive, evidenzbasierte Transfusions- } \\
\text { kriterien, patientenspezifische Prozedere } \\
\text { planen, Optimierung der physiologischen } \\
\text { Reserven und Risiken }\end{array}$ \\
\hline \multirow{2}{*}{ intraoperativ } & $\begin{array}{l}\text { elektive OP an individuelle } \\
\text { Gegebenheiten anpassen }\end{array}$ & $\begin{array}{l}\text { akribische Blutstillung, schonende OP-Technik, } \\
\text { anästhesiologische Therapie, Cell-salvage, } \\
\text { Koagulopathie meiden }\end{array}$ & $\begin{array}{l}\text { Optimierung des Cardiac Output, der Venti- } \\
\text { lation und Oxygenierung, restriktive Trans- } \\
\text { fusionskriterien }\end{array}$ \\
\hline \multirow{2}{*}{ postoperativ } & $\begin{array}{l}\text { Anämietherapie fortsetzen, } \\
\text { Medikamenteninteraktio- } \\
\text { nen vermeiden }\end{array}$ & $\begin{array}{l}\text { postoperatives Monitoring, sekundäre Blutun- } \\
\text { gen und iatrogene Blutverluste meiden, Nor- } \\
\text { mothermie, Infektvermeidung, ggf. Infektthe- } \\
\text { rapie, Medikamenteninteraktionen vermeiden }\end{array}$ & $\begin{array}{l}\text { Anämietoleranz optimieren, Anämiethera- } \\
\text { pie, Sauerstoffgabe optimieren, -verbrauch } \\
\text { minimieren, Infektvermeidung, ggf. Infekt- } \\
\text { therapie, restriktive Transfusionskriterien }\end{array}$
\end{tabular}

elektivem Hüftgelenksersatz [35]. In ausgewählten Fällen kommen zudem autologe Blutspenden infrage [7]. Im Rahmen von PBM-Programmen empfehlen Experten $[18,32]$, insbesondere bei komplexen Eingriffen bereits präoperativ die operative Vorgehensweise detailliert zu planen, den zu erwartenden Blutverlust abzuschätzen, die patientenindividuelle kardiale Kompensationsreserve zu ermitteln und die kritische Hämoglobingrenze festzulegen (Tab. 1).

Nach entsprechender präoperativer Vorbereitung stehen intraoperativ eine strikte Anwendung blutsparender Operationstechniken, eine akribische Blutstillung, ggf. eine maschinelle Autotransfusion, eine frühzeitige hämostaseologische Therapie und eine Optimierung der Herz-Kreislauf-Funktion sowie der Ventilation und Oxygenierung im Vordergrund [7, 18, 32,36].

Nach dem Eingriff sind ggf. Nachblutungen sofort chirurgisch anzugehen, die Normothermie anzustreben, das Sauerstoffangebot zu optimieren, postoperative Infektionen frühzeitig zu diagnostizieren und $\mathrm{zu}$ therapieren $[18,32]$. Insbesondere sind postoperative Anämien im Sinne einer konsequenten Transfusionsvermeidung oder Transfusionsreduktion stations- und fächerübergreifend z. B. durch Eisengaben fortgesetzt zu therapieren.

\section{Transfusionstrigger}

Die Querschnittsleitlinien der Bundesärztekammer zur Therapie mit Blutkomponenten und Plasmaderivaten unterscheiden bei ihren Angaben zu evidenzbasierten Transfusionstriggern grundsätzlich zwischen der

Tab. 2 Transfusionsempfehlungen: Erythrozyten bei akuter Anämie (nach [20]).

\begin{tabular}{|c|c|c|}
\hline Hb-Bereich & Kompensationsfähigkeit/Risikofaktoren & Transfusion \\
\hline$\leq 6 \mathrm{~g} / \mathrm{dl}(\leq 3,7 \mathrm{mmol} / \mathrm{l})$ & - & ja \\
\hline $\begin{array}{l}>6-8 \mathrm{~g} / \mathrm{dl} \\
(3,7-5,0 \mathrm{mmol} / \mathrm{l})\end{array}$ & Kompensation adäquat, keine Risikofaktoren & nein \\
\hline $\begin{array}{l}>6-8 \mathrm{~g} / \mathrm{d} \\
(3,7-5,0 \mathrm{mmol} / \mathrm{l})\end{array}$ & $\begin{array}{l}\text { Kompensation eingeschränkt, Risikofaktoren } \\
\text { vorhanden (z. B. KHK, Herzinsuffizienz, zerebro- } \\
\text { vaskuläre Insuffizienz)/Hinweise auf anämische } \\
\text { Hypoxie (physiologische Transfusionstrigger) }\end{array}$ & ja \\
\hline $\begin{array}{l}8-10 \mathrm{~g} / \mathrm{dl} \\
(5,0-6,2 \mathrm{mmol} / \mathrm{l})\end{array}$ & $\begin{array}{l}\text { Hinweise auf anämische Hypoxie (physiologische } \\
\text { Transfusionstrigger), bei ungestillter Blutung, } \\
\text { unklarer Klinik }\end{array}$ & ja \\
\hline$>10 \mathrm{~g} / \mathrm{dl}(\geq 6,2 \mathrm{mmol} / \mathrm{l})$ & - & nein \\
\hline
\end{tabular}

Tab. 3 Physiologische Transfusionstrigger (nach [20]).

\begin{tabular}{ll} 
Klinische Symptome & Tachykardie, Hypotension oder Blutdruckabfall, Dyspnoe \\
\hline EKG & neu auftretende ST-Senkungen/-Hebungen oder Rhythmusstörungen \\
\hline Echokardiografie & neu auftretende myokardiale Kontraktionsstörungen \\
\hline $\mathrm{O}_{2}$-Versorgung & - Anstieg der globalen $\mathrm{O}_{2}$-Extraktion $>50 \%$ \\
& - Abfall der $\mathrm{O}_{2}$-Aufnahme $>10 \%$ vom Ausgangswert \\
& - Abfall der gemischtvenösen $\mathrm{O}_{2}$-Sättigung $<50 \%$ \\
& - Abfall des gemischtvenösen $\mathrm{PO}_{2}<32 \mathrm{mmHg}$ \\
& - Abfall der zentralvenösen $\mathrm{O}_{2}$-Sättigung $<60 \%$ \\
\hline Laktazidose & Laktat $>2$ mmol/I + Azidose
\end{tabular}

- akuten, ungestillten Blutung oder der „unübersichtlichen“ klinischen Situation

- und der klinisch stabilen Situation ohne aktuellen Blutverlust und geben zusätzlich zu Hämoglobingrenzwerten auch klinische Parameter als sog. „physiologische Transfusionstrigger" an [20].
Im Fall der klinisch stabilen Situation ohne (Verdacht auf) ungestillte Blutung wird man Unfallopfer ohne weitere Begleitdiagnosen (z. B. koronare Herzkrankheit, Herzinsuffizienz, zerebrovaskuläre Insuffizienz) und Risikofaktoren erst bei Hämoglobinwerten unter $6 \mathrm{~g} / \mathrm{dl}$ transfundieren. Finden sich jedoch die Zeichen der „physiologischen Transfusionstrigger" oder liegen Begleitdiagnosen/Risikofaktoren vor, sind auch nach Unterschreiten eines Hämoglobinwerts von $8 \mathrm{~g} / \mathrm{dl}$ Erythrozytenkonzentrate zu verabreichen (Tab. 2). 
Tab. 4 Therapieindikationen für therapeutisches Plasma (nach [20]).

\begin{tabular}{|c|c|}
\hline $\begin{array}{l}\text { Indikation: } \\
\text { Störung der } \\
\text { Gerinnung }\end{array}$ & $\begin{array}{l}\text { - Anhebung der Gerinnungsfaktor- und Inhibitorspiegel bei komple- } \\
\text { xen Koagulopathien wegen manifester oder drohender schwerer } \\
\text { Blutungen } \\
\text { - Anhebung der Spiegel der Gerinnungsfaktoren V und XI oder der } \\
\text { Von-Willebrand-Faktor-Cleaving-Protease (Synonym: ADAMTS13), } \\
\text { weil für diese Therapien keine zugelassenen Einzelfaktorpräparate } \\
\text { zur Verfügung stehen }\end{array}$ \\
\hline $\begin{array}{l}\text { Indikation: } \\
\text { massive Blutung }\end{array}$ & $\begin{array}{l}\text { - kontinuierlicher Blutverlust }>100 \mathrm{ml} / \mathrm{min} \\
\text { - Substitutionsbedarf }>2 \text { Erythrozytenkonzentrate/15 min } \\
\text { - nach Transfusion von } \geq 4-6 \text { Erythrozytenkonzentraten } \\
\text { - bei drohenden mikrovaskulären Blutungen (mit Quickwert < } 50 \% \\
\text { oder APTT }>45 \text { s und/oder Fibrinogenspiegel }<1 \mathrm{~g} / \mathrm{l} \text { ) }\end{array}$ \\
\hline Dosierung & $\begin{array}{l}15-20 \mathrm{ml} \text { Plasma/kgKG und mit einer Geschwindigkeit von 30-50 ml/ } \\
\text { min zu verabreichen }\end{array}$ \\
\hline keine Indikation & $\begin{array}{l}\text { - reine Prophylaxe bei disseminierter intravasaler Gerinnung } \\
\text { - primärer Volumenersatz, parenterale Ernährung, Substitution von } \\
\text { Immunglobulinen } \\
\text { - Mangel von Gerinnungsfaktoren und Inhibitoren, die mit Einzelfak- } \\
\text { torkonzentraten wirksamer behandelt werden (z. B. Hämophilie A } \\
\text { und B, schwere cumarininduzierte Blutung, Ausnahme: Notfälle bei } \\
\text { fehlender rechtzeitiger Verfügbarkeit von Konzentraten oder Pro- } \\
\text { thrombinkomplex) }\end{array}$ \\
\hline
\end{tabular}

Tab. 5 Therapieindikationen für Thrombozytenkonzentrate (nach [20]).

\begin{tabular}{|c|c|}
\hline $\begin{array}{l}\text { allgemeine } \\
\text { Indikation }\end{array}$ & $\begin{array}{l}\text { - meist bei Massivtransfusionen } \\
\text { - selten bei Störung der Thrombozytenbildung oder bei vermehrtem } \\
\text { Thrombozytenverbrauch }\end{array}$ \\
\hline $\begin{array}{l}\text { Indikation bei } \\
\text { akuter Blutung }\end{array}$ & 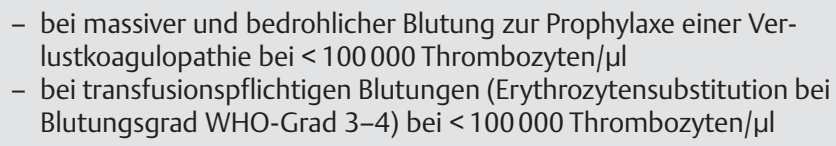 \\
\hline $\begin{array}{l}\text { Indikation vor } \\
\text { Elektiv-OP }\end{array}$ & $\begin{array}{l}\text { - präoperativ bei Patienten ohne zusätzliche Blutungsrisiken: } \\
\text { ab Thrombozytenzahl < } 50000 / \mu l \\
\text { - vor Operationen mit geringem Blutungsrisiko, bei denen eine Blut- } \\
\text { stillung durch Kompression erreicht werden kann: Thrombozyten- } \\
\text { zahlen } 20000-50000 / \mu l \\
\text { - präoperative Blutungsneigung und/oder Thrombozytenzahl } \\
\text { < } 20000 / \mu l: \text { präoperative Thrombozytengabe } \\
\text { - vor Eingriffen mit einem besonders hohen Blutungsrisiko (z. B. } \\
\text { neurochirurgische Eingriffe): präoperativer Thrombozytenwert } \\
\text { > } 70000 \text { bis } 100000 / \mu l \text { empfohlen }\end{array}$ \\
\hline
\end{tabular}

Die in Tab. 3 aufgelisteten „physiologischen Trigger" ([20], Kapitel 1.5.1.2) sollten in der klinischen Anwendung mehr Aufmerksamkeit erfahren.

Im Ausnahmefall besteht auch eine Transfusionsindikation, wenn bei Hämoglobinwerten von 8-10 g/dl Zeichen der „physiologischen Transfusionstrigger“" auftreten und/oder Begleitdiagnosen hierzu begründet Anlass geben; dies ist vornehmlich bei älteren, vorerkrankten Patienten mit geringer kardialer Kompensation gegeben. Oberhalb von $10 \mathrm{~g} /$ dl ergibt sich nur sehr selten eine Transfusionsindikation (Tab. 2).
„Hämoglobinkonzentrationen im Bereich von $10 \mathrm{~g} / \mathrm{dl}$ (6,2 mmol/l, Hämatokrit $30 \%$ ) anzustreben“ [20]. Dies gilt gleichermaßen für die prä-, intra- und postoperative Situation wie auch für den akut aufgenommenen polytraumatisierten Notfallpatienten, bspw. mit beginnender traumainduzierter Verbrauchskoagulopathie. Zusätzlich ist bei massivem Blutverlust eine frühzeitige Umstellung auf eine $1: 1: 1$-Ratio für Erythrozyten, Plasma und Thrombozyten [37] und eine hämostaseologische Intervention (z.B. Tranexamsäure) zu erwägen.

Eine Therapie mit gefrorenem Frischplasma ist gemäß Querschnittsleitlinien nur in einigen Fällen angezeigt, die in Tab. 4 zusammengefasst sind. Dabei erhöht $1 \mathrm{ml}$ Plasma die Gerinnungsfaktorspiegel um ungefähr $1 \mathrm{IE} / \mathrm{dl}$ bzw. den Quickwert um 1\% pro Kilogramm Körpergewicht, hingegen bei Umsatzsteigerung, z.B. bei Verbrauchskoagulopathie, nur um 0,5-1,0 IE/dl bzw. um 0,5-1,0\% und den Fibrinogenspiegel sogar nur um 0,02-0,03 g/l [20]. Die empfohlenen Dosierungen und zugehörigen Kontraindikationen sind Tab. 4 zu entnehmen.

Thrombozytenkonzentrate werden in der Unfallchirurgie/Orthopädie vornehmlich bei Massivtransfusionen, selten bei Störung der Thrombopoese oder bei vermehrtem Thrombozytenverbrauch substituiert [20]). Tab. 5 fasst die Indikationen zur Thrombozytentransfusion bei akuten Blutungen (ungestillte Blutung bzw. klinisch unklare Situation prä-, intra- und postoperativ) und für die elektive chirurgische Situation ohne ungestillte Blutung zusammen.

\section{Eigene Erfahrungen am Universitätsklinikum Münster}

Am UKM wurden im Jahr 2012 eine PBM-Initiative gestartet und als 1. Schritt ein regelmäßiger Blutverbrauchsbericht implementiert, der neben der sachlichen Informationsweitergabe auch das Bewusstsein für die Thematik („awareness") beim Adressaten schärfen soll. Hierzu werden fallbezogen für alle stationären Patienten die Verbräuche von Erythrozytenkonzentraten, gefrorenen Frischplasmen, Thrombozytenkonzentraten und plasmatischen Gerinnungsfaktoren erfasst. Auf dieser Basis wird regelmäßig für alle Kliniken ein Diagnosen- bzw. Prozeduren-bezogenes „Blutverbrauchsmonitoring“ einschließlich der innerbetrieblich entstehenden Kosten erstellt. Die Ausweitung auf ambu- 
lante Patienten befindet sich in der Vorbereitung. Neben den EDV-technischen Voraussetzungen mit Zugangsmöglichkeit für alle transfundierenden Klinikeinheiten ist die möglichst fehlerfreie und lückenlose Dokumentation aller Transfusionen von zentraler Bedeutung.

Vonseiten der Transfusionsmedizin werden für 6 Kliniken mit hoher transfusionsmedizinischer Relevanz - darunter Unfallchirurgie und Orthopädie - halbjährlich die diagnosenbezogenen Verbräuche aller Blutkomponenten klinikspezifisch zusammengestellt und in einer Trendanalyse z.B. mit der Gesamtfallzahlentwicklung und dem Case-MixIndex korreliert. Für die im „ranking“ nachfolgenden 10 Kliniken mit niedrigerer Transfusionsquote werden solche Aufstellungen jährlich generiert. Dabei werden die einzelnen Fälle in hausintern etablierten sog. klinischen Leistungsgruppen $[6,38,39]$ zusammengefasst, die sich deutlich besser als die G-DRGs an der klinischen Systematik und der Versorgungsrealität orientieren. Selbstverständlich lassen sich die Daten bis auf die Einzelfallebene differenziert darstellen, so z. B. für Massivtransfusionen, Faktor-VIIa-Behandlungen oder Patienten mit bekannter Hämophilie. Darüber hinaus sind auch stationsspezifische Auswertungen möglich. Die Halbjahresbzw. Jahresstatistik wird gemeinsam mit dem jeweiligen Klinikdirektor und Transfusionsbeauftragten der Klinik erörtert und ggf. auf Handlungsbedarf hin (z.B. geringere Bereitstellung von Blutprodukten präoperativ, Überdenken der Transfusionsregimes bei bestimmten $\mathrm{Di}$ agnosen/Prozeduren, spezifischer Schulungsbedarf für Ärzte/Pflegekräfte einer Klinik etc.) analysiert. Im nächsten Bericht wird dann der jeweilige Erfolg der bis dahin getroffenen qualitätssichernden Maßnahmen im Sinne eines PDCAZyklus erfasst.

Gemäß den Erfordernissen aus dem Transfusionsgesetz führt der Qualitätsbeauftragte für Hämotherapie zusammen mit Experten aus dem Bereich der Transfusionsmedizin krankenhausinterne Audits zur praktischen Umsetzung der Vorgaben aus den jeweils aktuellen Richtlinien und Querschnittsleitlinien sowie aus der klinikinternen Transfusionsvorschrift in allen transfundierenden Abteilungen durch und erstellt dazu regelmäßig Auditberichte für die jeweilige Klinik und den Klinikvorstand. Dabei werden ggf. auch Mängel erfasst (z.B. Aufklärungslücken, Dokumentations- ungenauigkeiten, Fehler bei der Transfusionsdurchführung oder beim Dokumentieren der Transfusionsindikation und des Transfusionserfolgs) und Maßnahmen zur Mängelbeseitigung und Qualitätsverbesserung kollegial vereinbart.

Im Rahmen unserer PBM-Initiative wird berufsgruppenübergreifend allen neuen Klinikmitarbeitern, die im Arbeitsalltag mit Transfusionen in Berührung kommen, direkt bei Dienstantritt eine 90-minütige Pflichtschulung zuteil. Hierbei wird sowohl die Idee des PBM, durch geeignete Maßnahmen allogene Bluttransfusionen weitgehend $\mathrm{zu}$ vermeiden, als auch das Ziel des „optimal blood use“, wenn eine Bluttransfusion medizinisch indiziert ist, intensiv vermittelt. Ziel ist stets die Transfusion der richtigen Blutkomponente für den richtigen $\mathrm{Pa}$ tienten zum richtigen Zeitpunkt.

Ergänzend werden Hilfestellungen zur praktischen Durchführung von Transfusionen (Zuständigkeiten, Verantwortlichkeiten und Kooperation der Berufsgruppen, Laborvoraussetzungen, zeitliche Abläufe, Verfügbarkeit und Bestellen von Produkten, Durchführung des Bedside-Tests und der Transfusion, Besonderheiten bei Notfall- und Massivtransfusionen, richtiger Umgang mit transfusionsassoziierten Risiken und Zwischenfällen, Kostentransparenz) gegeben. Für alle transfundierenden Ärzte und Pflegekräfte im „Klinikbestand“ werden zudem mind. jährlich transfusionsmedizinische Schulungen zur Auffrischung und Vertiefung auf freiwilliger Basis angeboten.

Durch diesen im Rahmen unserer PBMInitiative systematisch erstellten Maßnahmenkatalog (regelmäßige PBM-Berichte, Auditierungen, standardisierte Schulungen) wird die Universitätsklinik Münster ihrer Verantwortung bei der kontinuierlichen Verbesserung des Transfusionswesens und der Patientenbehandlung (Ergebnisqualität) gerecht.

\section{Fazit und Ausblick}

Neben den aktuellen Richtlinien zur Gewinnung von Blut und Blutbestandteilen und zur Anwendung von Blutprodukten sowie den Querschnittsleitlinien zur Therapie mit Blutkomponenten und Plasmaderivaten finden neuerdings anästhesiologisch geprägte Initiativen zum PBM Beachtung. Diese erheben den Anspruch, primär ein patientenzentriertes Ziel zu verfolgen. Gleichermaßen nutzt auch die Transfusionsmedizin die Grundsätze evidenzbasierter Medizin und hat die interdisziplinär im Konsens erstellten Querschnittsleitlinien der Bundesärztekammer (Stand 2009) maßgeblich mitgeprägt. Die Diskussion über präventive Strategien zur Transfusionsvermeidung und über eine sorgfältige, patientenindividuelle Indikationsstellung (Risiko-Nutzen-Abwägung) ist zu begrüßen. PBM-Initiativen in Australien, Österreich und der Schweiz haben das Bewusstsein dafür geschärft, dass insbesondere allogene Erythrozytentransfusionen einen unabhängigen Risikofaktor für ein schlechteres Behandlungsergebnis („patient outcome“) darstellen und die Vermeidung unnötiger Transfusionen dringend geboten ist.

Es sollte zur alltäglichen klinischen Routine werden, die präventive Behandlung einer Eisenmangelanämie vor einem Elektiveingriff konsequent durchzuführen, den Einsatz von gewebeschonenden blutsparenden Operationstechniken und den Gebrauch von Blutrückgewinnungsverfahren („cell saver“) systematisch weiterzuentwickeln.

Nicht zuletzt ist eine optimierte Gesamtversorgung des Patienten, einschließlich eines zielorientierten Gerinnungsmanagements (z. B. Point-of-Care-Diagnostik) und der Beachtung weiterer wichtiger Rahmenbedingungen (Normovolämie, Normothermie, Vermeidung einer Azidose) anzustreben. Die patientenindividuelle Festlegung eines noch akzeptablen Blutverlusts bzw. eines kritischen Herzfrequenzwerts (kritischer Hämoglobinwert oder Zeichen einer anämischen Hypoxie) sind wichtige Zielgrößen.

\section{Literatur}

${ }^{1}$ Henseler 0 . Report on notifications pursuant to Section 21 TFG (German Transfusion Act) for 2010 and 2011. Im Internet: http://www. pei.de/EN/information/blood-supply/reports/report-blood-supply-2010-2011$21 \mathrm{tfg}$-content.html

2 Wells AW, Mounter PJ, Chapman CE et al. Where does blood go? Prospective, observational study of red cell transfusion in north England. BMJ 2002; 325: 803-806

3 Anderson SA, Menis M, O'Connell Ket al. Blood use by inpatient elderly population in the United States. Transfusion 2007; 47: 582-592

${ }^{4}$ Beguin C, Closon MC, Vandekerckhove P et al. Concentration of transfusion resources on a few pathologies and a few patients: analysis of the comprehensive in-hospital patient database. Transfusion 2007; 47: 217-227

5 Brokent-Raven BA, Janssen MP, van der Poel CL et al. The PROTON study: profiles of blood product transfusion recipients in the Netherlands. Vox Sang 2010; 99: 54-64

${ }^{6}$ Geißler RG, Franz D, Buddendick H et al. Retrospective Analysis of the Blood Component Utilization in a University Hospital of Maximum Medical Care. Transfus Med Hemother 2012; 39: 29-138 
7 Spahn DR. Anemia and patient blood management in hip and knee surgery. Anesthesiology 2010; 113: 482-495

8 Statistische Ämter des Bundes und der Länder. Demografischer Wandel in Deutschland, Heft 1, Bevölkerungs- und Haushaltsentwicklung im Bund und in den Ländern. 2011; 1: 8-17; 1: $21-27$

${ }^{9}$ Greinacher A, Fendrich K, Alpen U et al. Impact of demographic changes on the blood supply: Mecklenburg-West Pomerania as a model region for Europe. Transfusion 2007; 47: 395401

10 Greinacher A, Fendrich K, Brzenske R et al. Implications of demographics on future blood supply: a population-based cross-sectional study. Transfusion 2011; 51: 702-709

11 Ali A, Auvinen MK, Rautonen J. The aging population poses a global challenge for blood services. Transfusion 2010; 50: 584-588

12 Seifried E, Klueter H, Weidmann C et al. How much blood is needed? Vox Sang 2011; 100: 10-21

13 Saß AC, Wurm S, Ziese T. Alter = Krankheit? Gesundheitszustand und Gesundheitsentwicklung - Somatische und psychische Gesundheit. In: Karin Böhm, Statistisches Bundesamt; Clemens Tesch-Römer, Deutsches Zentrum für Altersfragen; Thomas Ziese, Robert Koch-Institut, Hrsg. Beiträge zur Gesundheitsberichterstattung des Bundes, Gesundheit und Krankheit im Alter. Eine gemeinsame Veröffentlichung des Statistischen Bundesamtes, des Deutschen Zentrums fü Altersfragen und des Robert Koch-Instituts. 2009; 41-44

14 Ehling M, Pötzsch O. Demographic Changes in Germany up to 2060 - Consequences for Blood Donation. Transfus Med Hemother 2010; 37: 131-139

15 Ritter S, Willand L, Reinhard B et al. Blutspendepopulation in Deutschland: Demographie und Spendeaktivität. Bundesgesundheitsbl Gesundheitsforsch - Gesundheitsschutz 2008; 51: 915-925

16 Henseler O, Heiden M, Haschberger B et al. Report on Notifications Pursuant to $\$ 21 \mathrm{Ger}$ man Transfusion Act for 2008 and 2009 . Transfus Med Hemother 2011; 38: 199-216

17 Waters $J H$. Trauma sugery and transfusion options. Int Trauma Care 2008; 18: 66-70

18 Shander A, Javidroozi M, Perelman $S$ et al. From bloodless surgery to patient blood management. Mt Sinai J Med 2012; 79: 56-65

19 Richtlinien zur Gewinnung von Blut und Blutbestandteilen und zur Anwendung von Blutprodukten (Hämotherapie) (2010). Im Internet: http://www.bundesaerztekammer de/downloads/RiliHaemotherapie2010.pdf

20 Querschnitts-Leitlinien (BÄK) zur Therapie mit Blutkomponenten und Plasmaderivaten. 4. Aufl. (2008). Im Internet: http://www.dgti.
de/docs/doclink/10145/Querschnittsleitlinie_ Blutkomponente_22092009.pdf

${ }^{21}$ Robert Koch-Institut, Arbeitskreis Blut. Homepage. Im Internet: http://www.rki.de/DE/ Content/Kommissionen/AK_Blut/ak_blut_ node.html

22 Paul-Ehrlich-Institut. Hämovigilanz-Bericht des Paul-Ehrlich-Instituts 2010 - Auswertung der Meldungen von schwerwiegenden Transfusionsreaqktionen nach §63c AMG. Im Internet: http://www.pei.de/DE/infos/ fachkreise/haemovigilanz/bericht-haemovigilanz/teaser-bericht-2010.html

23 Opelz G, Sengar DP, Mickey MR et al. Effect of blood transfusions on subsequent kidney transplants. Transplant Proc 1973; 5: 253259

24 Vamvakas EC, Blajchman MA. Transfusion-related immunomodulation (TRIM): an update. Blood Rev 2007; 21: 327-348

25 Gombotz H, Rehak PH, Shander A et al. Blood use in elective surgery: the Austrian benchmark study. Transfusion 2007; 47: 1468-1480

${ }^{26}$ Hogshire L, Carson JL. Red blood cell transfusion: what is the evidence when to transfuse? Curr Opin Hematol 2013; 20: 546-551

27 Musallam KM, Tamim HM, Richards T et al. Preoperative anaemia and postoperative outcomes in non-cardiac surgery: a retrospective cohort study. Lancet 2011; 378: 13961407

${ }^{28}$ Hébert PC, Wells G, Blajchman MA et al. A multicenter, randomized, controlled clinical trial of transfusion requirements in critical care. Transfusion Requirements in Critical Care Investigators, Canadian Critical Care Trials Group. N Engl J Med 1999; 340: 409-417

${ }^{29}$ Carson Jl, Terrin ML, Noveck H et al.; FOCUS Investigators. Liberal or restrictive transfusion in high-risk patients after hip surgery. $\mathrm{N}$ Engl J Med 2011; 365: 2453-2462

30 Yazer $M H$, Waters JH. How do I implement a hospital-based blood management program? Transfusion 2012; 52: 1640-1645

31 Shander A, Van Aken H, Colomina MJ et al. Patient blood management in Europe. $\mathrm{Br} \mathrm{J}$ Anaesth 2012; 109: 55-68

32 Spahn DR, Goodnough LT. Alternatives to blood transfusion. Lancet 2013; 381: 18551865

${ }^{33}$ Williamson LM, Devine DV. Challenges in the management of blood supply. Lancet 2013; 381: 1866-1875

${ }^{34}$ Anker SD, Colet JC, Filippatos G et al.; FAIR-HF Trial Investigators. Ferric carboxymaltose in patients with heart failure and iron deficiency. N Engl J Med 2009; 361: 2436-2448

35 Doodeman HJ, van Haelst IM, Egberts TC et al. The effect of a preoperative erythropoietin protocol as part of a multifaceted blood management program in daily clinical practice. Transfusion 2013; 53: 1930-1939
${ }^{36}$ Walsh TS, Palmer J, Watson D et al. Multicentre cohort study of red blood cell use for revision hip arthroplasty and factors associated with greater risk of allogenic blood transfusion. Br J Anaesth 2012; 108: 63-71

37 Cotton BA, Gunter OL, Isbell J et al. Damage control hematology: the impact of a trauma exsanguination protocol on survival and blood product utilization. J Trauma 2008; 64: 1177-1183

${ }^{38}$ Roeder N, Siebers L, Frie M et al. DRG-Akzeptanz verbessern - Kliniker erreichen mit klinischen Leistungsgruppen. Das Krankenhaus 2006; 5: 390-401

${ }^{39}$ Helling J, Bunzemeier H, Fiori $W$ et al. Klinische Leistungsgruppen - Update und Ausblick. Das Krankenhaus 2009; 9: 857-862

\section{Priv.-Doz. Dr. med. Georg Geissler} Facharzt

Prof. Dr. med. Peter Schlenke

Leitender Oberarzt

Prof. Dr. med. Dr. rer. nat.

Walter Sibrowski

Institutsdirektor

Universitätsklinikum Münster Institut für Transfusionsmedizin und Transplantationsimmunologie Albert-Schweitzer-Campus 1

Gebäude D11

48149 Münster

Medizinische Universität Graz

Universitätsklinik für Blutgruppenserologie und Transfusionsmedizin Auenbruggerplatz 3

8036 Graz

Österreich

georg.geissler@ukmuenster.de

\section{Dr. med. Clemens Kösters Oberarzt}

Prof. Dr. med. Michael J. Raschke Klinikdirektor

Universitätsklinikum Münster Klinik für Unfall-, Hand- und Wiederherstellungschirurgie Albert-Schweitzer-Campus 1 Gebäude W1 48149 Münster 